\title{
Mitochondria: The Secret Chamber of Therapeutic Targets for Age-Associated Degenerative Diseases
}

\author{
Youngmi Kim PAK ${ }^{1, *}$, and Jae Hoon JEONG ${ }^{2}$ \\ ${ }^{1}$ Department of Physiology, College of Medicine, ${ }^{2}$ Department of Life and Nanopharmaceutical Science, \\ College of Medicine, Kyung Hee University, Seoul 130-701, Republic of Korea
}

(Received July 12, 2010; Accepted July 16, 2010)

\begin{abstract}
Mitochondria have long been recognized as ATP engines for the cell and recently as a dynamic and mobile organelles that control cell death and life. This exquisite organelle is the site of reactive oxygen species production and is highly vulnerable to exogenous stresses, resulting in catastrophic damage to the cell. Mitochondrial dysfunction is linked to a wide range of age-associated degenerative diseases, such as metabolic syndrome, cardiovascular disease, and neurodegenerative diseases. Understanding the molecular mechanisms of mitochondria-dependent pathogenesis may provide important strategies to treat these diseases. Indeed, mitochondria are emerging therapeutic targets for the mitochondria-related diseases. In this paper, we review the recent concepts of mitochondrial biology and how mitochondria are involved in ageassociated degenerative diseases. Furthermore, we summarize the therapeutics which target to improve mitochondrial functions.
\end{abstract}

Keywords: Mitochondria, Degenerative disease, Therapeutic, Mitochondrial medicine

\section{INTRODUCTION}

Mitochondria are dynamic organelles essential for cellular life, death and differentiation. Although mitochondria are best known for ATP production via oxidative phosphorylation (OXPHOS), they are also critical for ion homeostasis, apoptosis and biochemical metabolic pathways including Krebs cycle, $\beta$-oxidation, and lipid and cholesterol synthesis. Given their fundamental roles in the human body, mitochondrial deficiency can theoretically give rise to any symptom, in any organ, at any age; thus mitochondria are considered as a "powerhouse of disease" (Lane, 2006a, b). Indeed, mitochondrial biology is one of the fastest growing research areas in genetics and medicine because mitochondrial dysfunctions play a role not only in rare childhood genetic diseases but also possibly in many common age-associated diseases, including metabolic syndrome, neurodegenerative diseases, cardiovascular diseases, and cancer. Although it had been difficult to under-

${ }^{*}$ Corresponding author

Tel: +82-2-961-0908 Fax: +82-2-969-6343

E-mail: ykpak@khu.ac.kr stand whether a mitochondrial defect in cells is a cause or an effect of the diseases, it is now generally accepted that mitochondrial degeneration causes these age-associated diseases. However, we still do not understand what causes mitochondria dysfunctional and how to prevent or cure these diseases. In this review, we cover the modern mitochondrial biology from basic mitochondrial pathogenesis to recent concepts for mitochondrial therapeutics.

\section{MITOCHONDRIAL BIOLOGY}

\section{An organelle encoded by two genomes}

Mitochondria are the only cellular organelles having their own DNA, mitochondrial DNA (mtDNA), which is distinct from the nuclear DNA (nDNA). Approximately 1001,000 copies of closed-circular, double-stranded mtDNAs are present in each cell; however, the numbers vary depending on cell types. Human mtDNA (16,569 bp) contains only 37 genes, encoding 13 subunits of OXPHOS, 12S and 16S rRNA, and 22 tRNAs (Wallace, 2005; Schapira, 2006). Since the mtDNA-encoded basic machinery for protein synthesis is used to synthesize 13 polypeptides in mi- 


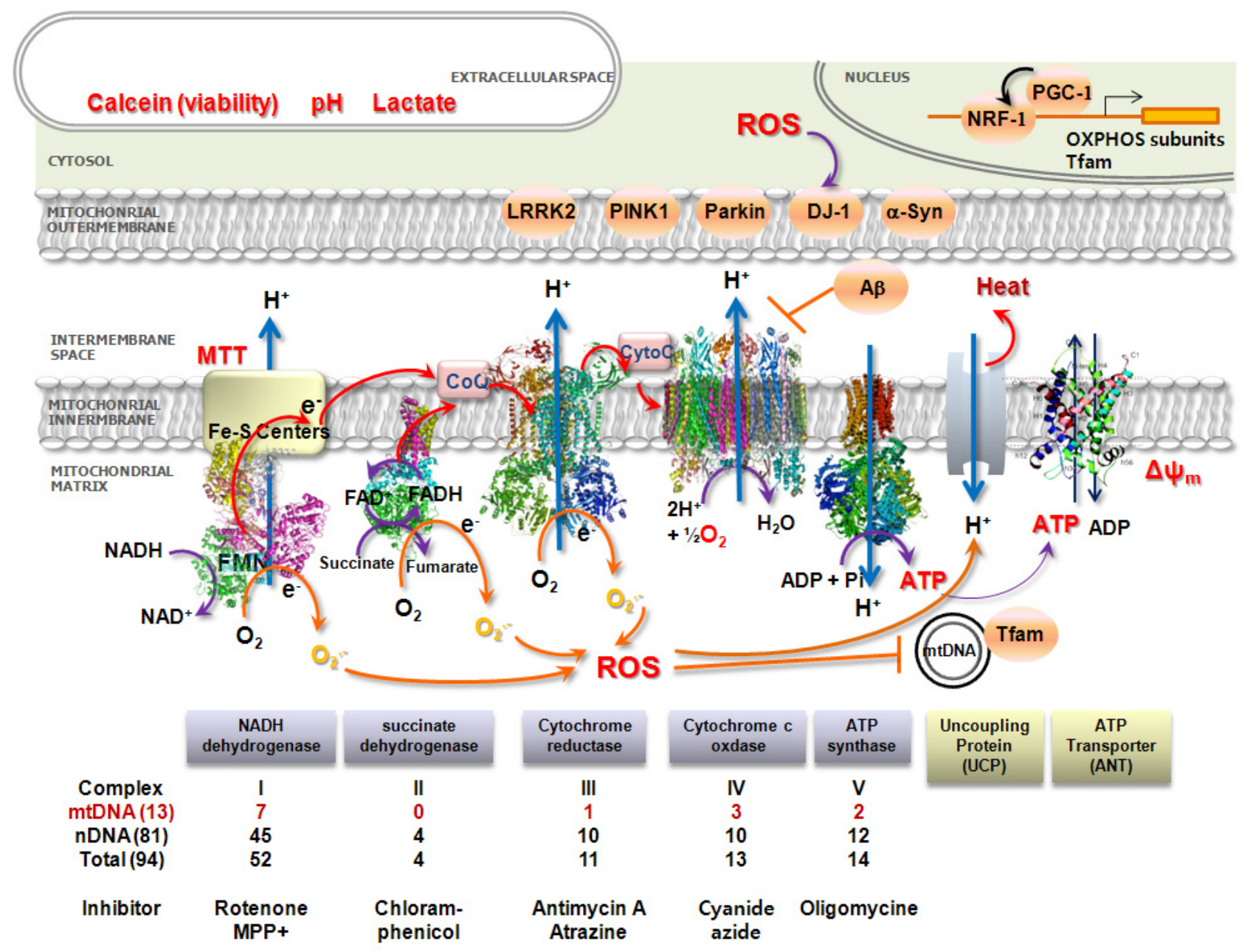

Fig. 1. Electron transfer system (ETS) and reactive oxygen species (ROS) production in mitochondria. Electrons from NADH and succinate $\left(\mathrm{FADH}_{2}\right)$ pass through complex I, II and III via coenzyme Q (Q). Then, cytochrome c (cyto c) transfers e- to complex IV, which is coupled to ATP synthase (complex V) to generate ATP. Oxidative phosphorylation (OXPHOS) covers both ETS and ATP synthase. Flow of $\mathrm{e}^{-}$is followed by proton $\left(\mathrm{H}^{+}\right)$pumping across the inner membrane, generating mitochondrial membrane potential $\left(\Delta \psi_{\mathrm{m}}\right)$. Uncoupling protein (UCP) uncouples complex IV and $\mathrm{V}$ and pumps protons into matrix to produce heat. ATP/ADP transporters (adenine nucleotide transporter, ANT) exchange ATP and ADP across the inner membrane to supply ATP to cytosol and ADP to mitochondria. Electrons leaked from complexes I, II, and III react with $\mathrm{O}_{2}$ to produce ROS. Generally, anti-oxidant systems in mitochondria remove ROS. The mtDNA-encoded and nDNA-encoded subunits and specific inhibitors of OXPHOS complexes are summarized at the bottom of figure. The genes/proteins in orange balls are involved in either mitochondria control or disease development; TFAM, NRF-1, PGC-1, LRRK2, PINK1, Parkin, DJ-1, $\alpha$-synuclein ( $\alpha$-Syn), and amyloid $\beta$ (A $\beta$ ). PGC-1 co-activates NRF-1 in transactivation of TFAM and nDNA-encoded OXPHOS subunits in nucleus. Red letters are the parameters analyzed in CMAPS assay; calcein (viability), $\mathrm{pH}$ and lactate concentration in extracellular space, MTT, membrane potential ( $\Delta \psi_{\mathrm{m}}$ ), ATP contents, $\mathrm{O}_{2}$ consumption rate (OCR), ROS, and mRNAs for mtDNA-encoded 13 subunits.

tochondria, functional mitochondria require other nDNAencoded proteins involved in mitochondrial replication, transcription, and translation (see Fig. 1 for summary). In fact, $-1,500-2,000$ mitochondrial proteins, excluding 13 mtDNA-encoded proteins, are translated on cytosolic ribosomes and post-translationally imported into mitochondria.

MtDNA shows a few distinct characteristics compared to nDNA. First, mtDNA is more susceptible to mutation than
nDNA, because it is located proximal to reactive oxygen species (ROS) generation (Madamanchi and Runge, 2007). The mutations in mtDNA accumulate with aging possibly because of increasing ROS (Cortopassi et al., 1992; Barja and Herrero, 2000; Chan, 2006a). Second, since there are many mtDNA molecules in a cell, each cell may contain either wild type or mutant mtDNAs, or both. The co-existence of wild type and mutant mtDNA is called "hetero- 
plasmy", while the existence of all identical mtDNAs is called "homoplasmy". Third, mtDNA is maternally inherited because no paternal mtDNA from the sperm enters the ovum during fertilization, and also because the ovum could degrade any paternal mtDNA that may accidently enter the ovum. Fourth, the transcriptional products of mtDNA (mtRNA) are polycistronic and contain no intronic regions; features that are similar to those of prokaryotes. These characteristics of mtRNA give a rationale to propose the "symbiosis theory" of mitochondria.

\section{Cell-based mitochondrial activity profiling system (CMAPS)}

The numbers of OXPHOS subunits encoded by mtDNA or $\mathrm{nDNA}$ are summarized under each complex of the mitochondrial electron transfer system (ETS) and ATP synthase (OXPHOS system) in Fig. 1. No subunit of complex II (succinate dehydrogenase, SDH) is encoded by mtDNA, thus mitochondrial damage often increases SDH activity and expressions to restore the mitochondrial function. Therefore, a systemic mitochondrial function analysis is required to monitor whether mitochondria of cells or tissues are indeed damaged or activated by stresses or drugs. Instead of using isolated mitochondria, a cell-based mitochondrial activity analysis profiling system (CMAPS) was adapted to assay overall mitochondrial functions in our laboratory. CMAPS assay is similar to the large scale screening compendium using a 384-well plate reported by another group (Wagner et al., 2008), except that we used 96well plates. In CMAPS, we measured cell viability using calcein dye and monitored mitochondrial physiology related to OXPHOS. These include the TMRE (tetramethylrhodamine ethylester) assay which measures mitochondrial membrane potential $\left(\Delta \psi_{\mathrm{m}}\right)$; the MTT ((3-(4,5-dimethylthiazol-2-yl)-2,5-diphenyltetrazolium bromide)) assay, which measures mitochondrial dehydrogenase (complex I) activity; a luciferase-based intracellular ATP assay; a ROS assay using fluorescent probe (CM- $\mathrm{H}_{2} \mathrm{DCFDA},(5-$ (and-6)chloromethyl-2', 7'-dichlorodihydrofluorescein diacetate); and an assay for endogenous cellular oxygen consumption rate (OCR), which measures the cellular capacity to consume oxygen by complex IV (Lim et al., 2009). Abnormally increased production of ROS, a byproduct of OXPHOS complex I, II or III, may damage mtDNA in matrix as well as other proteins. The ROS-mediated mutations or deletions of mtDNA lead to decreased mtDNA copy numbers and/or mtRNAs reciprocally, and construct a vicious cycle of exacerbation of mitochondrial dysfunction.

\section{Regulation of mitochondrial biogenesis}

Cell-specific energetic, metabolic, and signaling demands finely control the abundance, morphology, and functional properties of mitochondria. This control is largely achieved at the level of transcription, in response to a wide range of physiological signals such as physical activity, nutrient availability, temperature, circadian cues, and exposure to infectious agents. The interconnected network of transcription factors regulates a broad set of nuclear genes encoding mitochondrial proteins (for example, nDNA-encoded OXPHOS subunits) as well as the controlling factors for replication and transcription of mtDNA, responding to physiological cues. Failure of the mitochondrial adjustments leads to many human diseases.

The D-loop region (-1.2 kb), a short three-strand structure of $m t D N A$, is the major site of mtDNA replication and transcription (Falkenberg et al., 2007). Several nDNA-encoded proteins are known for binding to D-loop regions and control mitochondrial biogenesis: mitochondrial transcription factor A (TFAM) (Larsson et al., 1998; Ekstrand et al., 2004), mitochondrial transcription factor B (TFBM) (Matsushima et al., 2004), mtDNA polymerase gamma (POLG) (Gaspari et al., 2004), and mitochondrial single-stranded DNA binding protein (mtSSB). Defects on these D-loop binding proteins also lead to mitochondrial damage and consequently to the diseases (Silva et al., 2000; Larsson and Rustin, 2001; Trifunovic et al., 2004).

TFAM is essential for mtDNA transcription and replication since TFAM is required for initiation of mtRNA transcriptions and TFAM-mediated mtRNA fragments are required for priming $\mathrm{mtDNA}$ replication on the D-loop region (Falkenberg et al., 2007). Obviously, TFAM knock-out induced mtDNA depletion and caused embryonic lethality or major cellular dysfunction (Larsson et al., 1998). The expression of TFAM is regulated by NRF-1/2, which are two major transacting factors (Choi et al., 2004). Peroxisome proliferator-activated receptor $\gamma$-coactivator (PGC)-1 $\alpha$, stimulates the expression of NRF-1 and coactivates the transcriptional activity of NRF-1 on the promoter of TFAM and other nDNA-encoded mitochondrial subunits of OXPHOS (see nucleus in Fig. 1) (Choi et al., 2006; Liang and Ward, 2006). PGC-1 $\alpha$ was identified as a thermogenesis regulator in brown fat (Puigserver et al., 1998) and is verified as an important stimulator of mitochondrial biogenesis. Thus, PGC-1 $\alpha$ null mice exhibited multi-system abnormalities ranging from reduced muscle and heart functions to obesity and hepatic steatosis, indicating disturbance of energy metabolism (Leone et al., 2005). Their phenotypes are similar to those of TFAM null mice (Larsson and Rustin, 2001). 


\section{Dynamic mitochondria with reticulum structure}

The mitochondrion is a highly dynamic organelle that is often organized as a continuous reticulum, but that can fragment into smaller tubular structures depending on the cellular states (Capaldi, 2000). Textbooks still describe mitochondria being as "small, approximately $1 \mu \mathrm{m}$ diameter, circular or cigar-shaped structures having an outer membrane, the folded inner membranes (cristae), and matrix", as first visualized by electron microscopy more than 50 years ago. However, this long-held picture is inadequate, since mitochondria form tubular structures and constantly is remodeled by both fission and fusion. In addition, they move along actin filaments (microtubule) via kinesin and dynein motors (Fig. 2). But the mitochondrial reticular morphology may be dependent on fission and fusion rather than cytoskeletal polymerization. The mitochondrial network may share solutes, metabolites, proteins, and electrochemical gradients with the neighbor, and the fission/fusion is a mechanism to complement a damaged unit and possibly recover its activity (Chan, 2006b).

A number of proteins involved in mitochondrial fission and fusion have been reported in yeast: Dmn1, Fis1, Mdv1/Caf4, Fzo1, Mgm1, and Ugo1 (Okamoto and Shaw, 2005; Hoppins et al., 2007). A three protein MMM complex
A

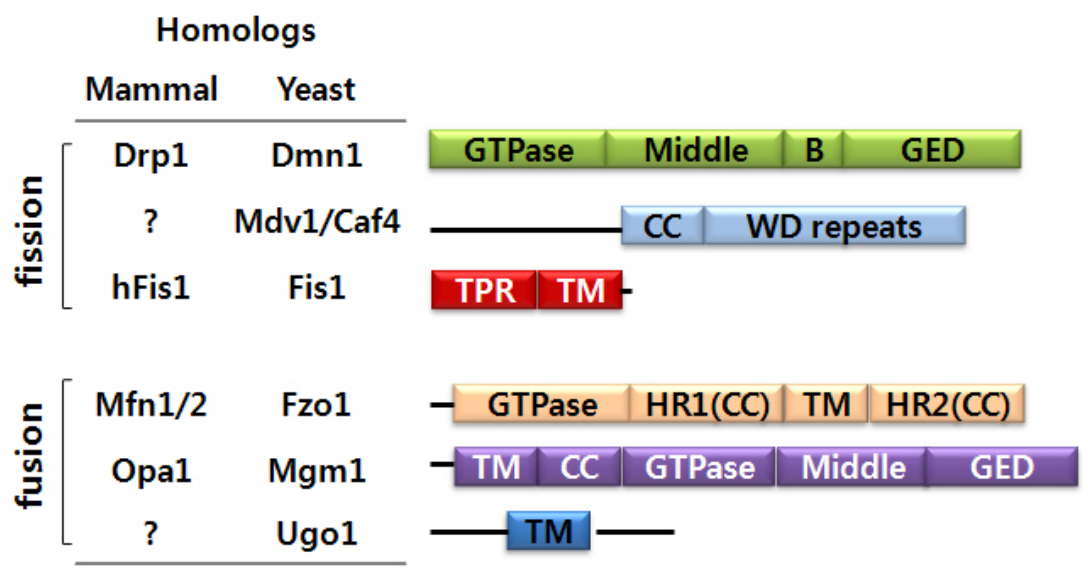

C

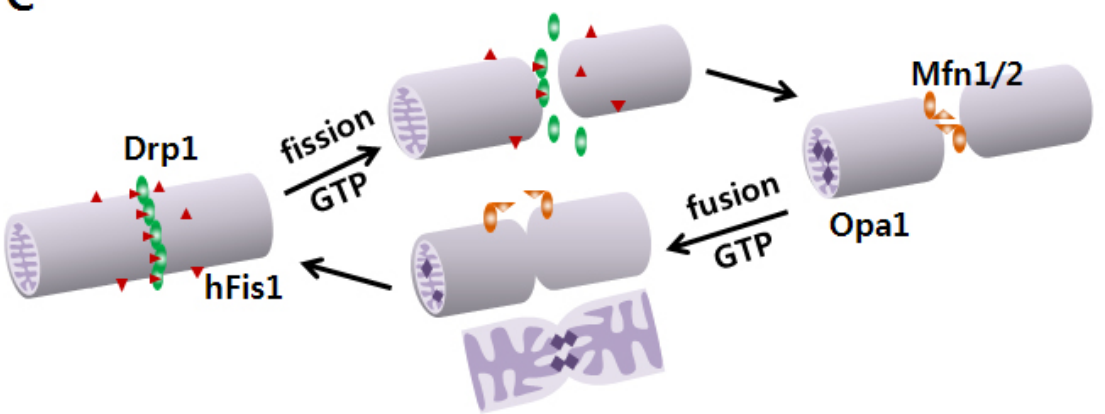

B
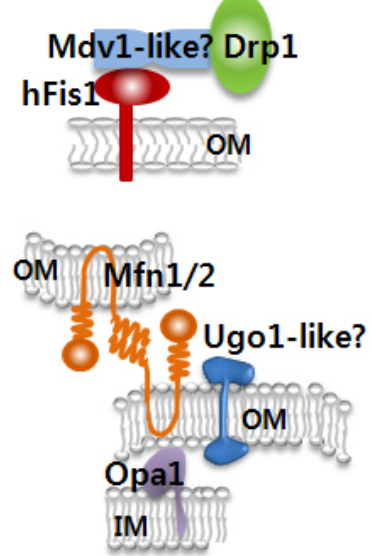

D

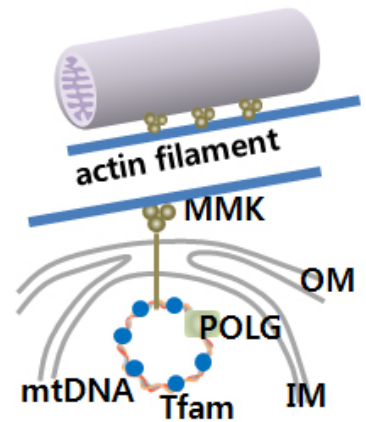

Fig. 2. The mammalian mitochondrial machinery for fission and fusion. (A) Schematic structures of human fission (Drp1 and hFis1) and fusion (Mfn1/2 and Opa1) proteins. Drp1. Mfn1/2, and Opa1 contain GTPase domains. Mfn1/2 and Opa1 contain hydrophobic heptad repeat (HR) coiled-coil (CC) regions. (B) Schematics of the steady-state localization of the mitochondrial fission (upper) and fusion (lower) proteins in mammalian cells. The transmembrane segments of the Mfns are unusually long and contain charged residues that are thought to allow a U-turn in the mitochondrial OM, so that almost the entire protein faces the cytosol. Spirals of Mfn $1 / 2$ represents coiled coil (CC) domain. Human homologs for Mdv-1 and Ugo1 are not yet identified. (C) A proposed mechanism of mitochondrial fission and fusion. (D) The proposed mitochondrial movement attaching kinesin complex on actin filament (upper) and the proposed anchoring of mtDNA replication foci with cytoskeletal elements at contact site of mitochondria (lower). The Miro/milton/kinesin (or dynein) triple complex (MMK) mediates mitochondrial transport by anchoring microtubules in neuronal cells. The simple mtDNA nucleoid complex of mtDNA, POLG (green), and Tfam (blue) is schematically shown. CC: coiled-coil, GED: GTPase effector domain, GTPase: GTPase domain, middle: middle domain, TM: transmembrane domain, OM: mitochondrial outer membrane, IM: mitochondrial inner membrane. 
(Mmm1p, Mdm10p, and Mdm12p) maintains mitochondrial tubular structure and may also link mtDNA nucleoids at a mitochondria contact site to the actin filament (Okamoto and Shaw, 2005). In mammals, only five proteins have been identified: dynamin-related protein 1 (Drp1) and hFis1, which are essential for mitochondrial fission, while mitofusin (Mfn)1/2 and Optic Atropy-1 protein (Opa1) are fusion proteins (Okamoto and Shaw, 2005; Chan, 2006b). Structures and hypothesized quaternary assemblies of the mitochondrial fission/fusion components are summarized in Fig. 2A and 2B, respectively. Drp1 is a large GTPase protein which is present in both cytosol and mitochondrial outer membrane and Fis 1 is a small protein that is uniformly localized in mitochondrial outer membrane. As mitochondria undergo fission, Drp1 binds to Fis1 at the initial constriction site in the middle of the mitochondrial tubule, and then divides mitochondrial reticulum using GTP. Knockdown of either Fis1 or Drp1 using siRNA resulted in formation of elongated mitochondria. On the other hand, homotypic interactions between hydrophobic heptad repeat $(\mathrm{HR})$, coiled coil (CC) domain, of two Mfn1 molecules on outer membrane lead to fusion of mitochondrial outer membranes (Fig. 2C). Opa1, which is present in the inner membrane, is a little complex and mediates the fusion of inner membranes. The siRNA knock down of Opa1 damages cristae structure and causes mitochondrial fragmentation (Chan, 2006b).

Mitochondria are also mobile organelles. Cells regulate mitochondrial movement depending on calcium signaling in order to meet the changing energy needs of each cellular region (Wang and Schwarz, 2009). Mitochondria are often transported considerable distance from cell body to the axon and dendrite (anterograde) and the reverse direction (retrograde) in neuron cells. The anterograde mitochondrial motility is regulated by kinesin motors, while the retrograde transport is mediated by dynein motors, which are connected to mitochondria adaptor proteins to form a Miro/milton/kinesin (MMK) complex (Fig. 2D, upper). Further studies are needed to determine whether the MMK complex is connected with mtDNA nucleoids like yeast MMM complex (Fig. 2D, lower).

The disruption of this mitochondrial dynamic/mobile equilibrium may cause cell injury or death and contribute to development of age-associated degenerative diseases. Mutations in the opa1 or mfn2 genes were identified in patients with autosomal dominant optic atrophy and CharcotMarie-Tooth neuropathy type $2 \mathrm{~A}$, respectively, providing direct evidence linking mitochondrial dynamics and human pathogenesis (Okamoto and Shaw, 2005). In non-genetic diseases, beta-amyloid protein-induced nitric oxide in- creased S-nitrosylation of Drp1 resulted in mitochondrial fission, possibly leading to Alzheimer's disease (Cho et al., 2009). Similarly, the disturbance of mitochondrial fission/fusion by Mfn1 or hFis1 impairs mitochondrial signal generation and insulin secretion in pancreatic beta cells (Park et al., 2008). The depolarized/damaged/fragmented mitochondria recruit parkin proteins to remove the dysfunctional mitochondria by autophagy (McBride, 2008; Narendra et al., 2008). In fact, the mitochondrial filament fusion after the fission very selectively segregates mitochondria to be removed by autophagy (Twig et al., 2008) and failure of this autophagy process may also lead to diseases (Chen and Chan, 2009; Fujitani et al., 2010). The cross- talk between fission and fusion reactions with transport machineries remains to be elucidated to understand their implications in the diseases (MacAskill and Kittler, 2010).

\section{MITOCHONDRIAL DISEASES}

Mitochondrial diseases are defined as diseases resulting from either inherited or spontaneous mutations in mtDNA or nDNA which lead to deleterious functions of the mitochondrial proteins or RNA molecules (Wallace, 2005; Schapira, 2006). Additionally, a wide range of age-associated disorders and various forms of cancer are also caused by mitochondrial stresses and environmental factors, such as nutrients and various other stresses which also induce mitochondrial damage (Lee et al., 2005; Lim et al., 2009). We defined the environmental factors that disrupt normal mitochondrial functions as mitochondrial stress. Environmental stress can result in ROS generation and inflammatory responses, contributing to impaired metabolism in mitochondria. The mitochondrial stress- inflammation loop can further promote inflammatory signaling and contribute to the metabolic deterioration that is associated with atherosclerosis, obesity, type 2 diabetes and neurodegenerative diseases, depending on the cell type involved.

\section{Aging, mitochondria, and ROS}

Aging is the greatest risk factor for degenerative diseases (Wallace, 2005; Yankner et al., 2008). It is well known that age-dependent accumulation of mtDNA mutations, especially large-scale deletions and point mutations, correlates with declines in mitochondrial function. (Michikawa et al., 1999; Wang et al., 2001; Larsson, 2010). The mitochondrial theory of aging proposes that the damaged mitochondria produce ROS with time, leading to increasing amounts of oxidative damage to various cell components. Regardless of insult types, mitochondrial stress can cause 
an imbalance between ROS production and removal, resulting in a net increase of ROS. The increases in ROS can lead to further mtDNA damage and destabilization of mitochondrial ROS homeostasis, thus completing a vicious cycle of ROS generation (Fig. 1). Oxidative damage by ROS plays a major role in cell death, which affects life span. On the other hand, the mtDNA polymerase- $\gamma$ (POLG) deficient mice demonstrated accumulation of mtDNA mutations without changing amounts of ROS (Trifunovic et al., 2005). This is due to lack of proofreading activity, resulting in decreased ATP production, tissue degeneration by increased apoptosis, and consequent early senescence of the mice (Trifunovic et al., 2004; Kujoth et al., 2005). Interestingly, transcriptional profiling of aged human brain revealed that gene expressions which are involved in mitochondria functions were decreased, followed by increased stress-response and antioxidant gene expressions (Lee et al., 2002). Thus, various disturbances of mitochondrial gene expression might be important for developing age-dependent chronic diseases.

\section{Metabolic syndrome}

Metabolic syndrome is a manifestation of one or more metabolic risk factors such as upper body obesity, atherogenic dyslipidemia, hypertension, and hyperglycemia, in one subject. Insulin resistance is characterized by a diminished ability of cells or tissues to respond to physiological levels of insulin. Genetic and environmental factors, including aging, obesity, lack of exercise, and stress are believed to contribute to insulin resistance via mitochondrial deterioration in various tissues. Therefore, the mitochondrial defect-mediated insulin resistance may be a common denominator in pathophysiology for many chronic diseases.

For the past 20 years, considerable evidence has been accumulating showing a direct association between mitochondrial function and insulin resistance. For example, epidemiological studies demonstrated that a decrease in mtDNA copy number occurs prior to the development of type 2 diabetes (Lee et al., 1998; Song et al., 2001) and that mtDNA copy number is negatively correlated with parameters of insulin resistance (Park et al., 1999). A study by our group proved that mtDNA-depletion-mediated mitochondrial damage caused the impairment of glucose metabolism and cellular glucose uptake (Park et al., 2001). This was the first evidence proving that mitochondrial disturbance is a cause, not an effect, of a metabolic disorder. Important progress towards showing a direct relationship between mitochondrial dysfunction and insulin resistance in humans was made by conducting an analysis using non-invasive in vivo ${ }^{13} \mathrm{C}-{ }^{31} \mathrm{P}$ NMR spectroscopy. Elderly subjects or the offspring of type 2 diabetic patients showing insulin resistance, exhibited a reduced mitochondrial OXPHOS activity of up to $40 \%$ (Petersen et al., 2003; Petersen et al., 2004). Microarrays of biopsy samples from human skeletal muscle revealed that expressions of PGC-1a- and NRF-1-dependent genes were reduced in insulin resistant and diabetic subjects (Patti et al., 2003). This study shows that the decreased mitochondrial gene expression may contribute to metabolic disturbances which are characteristic of insulin resistance and diabetes. In our laboratory, we have reported that G1444A single nucleotide polymorphism (SNP) in the PGC-1 $\alpha$ gene, which changes a glycine in codon 482 to serine, failed to co-activate TFAM transcription, resulting in a decrease of mtDNA copy number (Choi et al., 2006). These findings suggest that the activity or genetic variations of PGC- $1 \alpha$ may contribute to individual variations in mitochondrial function and insulin resistance or diabetes.

\section{Neurodegenerative diseases}

Neurodegenerative diseases are a heterogeneous group of disorders characterized by progressive and selective loss of neuronal cells. Despite the heterogeneity of neurodegeneration, mitochondria are likely to be involved as a common theme in the etiology of these diseases.

Alzheimer's disease (AD) is characterized by the presence of amyloid beta (AB) plaque and neurofibrillary tangles in brain. Recently, it was reported that $A \beta$ impaired the OXPHOS complex IV in the inter-membrane space of mitochondria (Fig. 1) to produce ROS (Lin and Beal, 2006). Also, $A \beta$ interacts with mitochondrial $A \beta$-binding alcohol dehydrogenase (ABAD), resulting in the inhibition of $A B A D$. Interaction between $A B A D$ and $A \beta$ causes mitochondrial stress and apoptosis (Lustbader et al., 2004; Schapira, 2006). Although the glial functions in the central nervous system are important in controlling neuroinflammation and neurodegeneration, the role of glial OXPHOS complex IV in inflammatory conditions is not known well.

The link between Parkinson's disease (PD) and mitochondria was first established with the identification of a deficiency in OXPHOS complex I activity in PD substantia nigra in designer-drug abusers. The chemical MPTP (1methyl 4-phenyl-1,2,3,6-tetrahydropyridine), and its metabolite $\mathrm{MPP}^{+}$, block complex I and was defined later as a inducer for PD animal models. However, the pathogenesis of sporadic PD, consisting of $90 \%$ of total PD, is not yet clear. Many genes implicated in PD are also associated with mitochondria. So far, mutations or polymorphisms in mtDNA and $15 \mathrm{nDNA}$-encoded genes have been identified as PD-linked genes, although these mutations are rare 
causes of PD. Of these, $\alpha$-synuclein ( $\alpha$-Syn), parkin, DJ-1, phosphatase and tensin homologue (PTEN)-induced kinase 1 (PINK1), and leucine-rich-repeat kinase 2 (LRRK2) are directly or indirectly involved in mitochondria. Especially, PINK1 mutations in familial PD, lose serine/threonine kinase activity in the mitochondrial membrane and PINK1 deficiency leads to mitochondrial abnormalities and dopaminergic neuron degeneration. PINK1 phosphorylates the downstream target protein, Parkin, in a fly model, possibly to maintain mitochondrial homeostasis and to protect neurons (Park et al., 2006). Parkin is also involved in mitochondrial dynamics, since it is selectively recruited to dysfunctional/fragmented mitochondria with low membrane potentials in mammalian cells. After recruitment, Parkin mediates the mitochondrial autophagy and the selective elimination of impaired mitochondria (Narendra et al., 2008). Therefore, Parkin mutation may fail to eliminate dysfunctional mitochondria leading to PD. Over-expression of disease-related LRRK2 mutants in C. elegans demonstrated that LRRK2 protects against mitochondrial dysfunction induced by rotenone (Saha et al., 2009).

Mutant huntingtin causes Huntington's disease (HD), an incurable disease manifested by involuntary movements and psychiatric disorder. The mutant huntingtin contains an abnormally expanded polyglutamine domain in striatal medium spiny GABAergic projection neurons and detrimentally impairs mitochondrial functions and calcium homeostasis, resulting in early degeneration and atrophy (Perry et al., 2010). The results show that HD may also be caused by mitochondrial dysfunctions.

\section{Cardiovascular diseases}

Cardiovascular disease is one of the major complications of diabetes and metabolic syndrome. Mitochondrial damage induced by ROS overproduction promotes atherosclerosis by increasing oxidation of low-density lipoprotein and injury of endothelial cells, which can result in myocardial infarction, stroke, and ischemic/reperfusion damage (Madamanchi and Runge, 2007). Besides ROS production, mitochondrial stresses, such as dideoxycystidine (a mtDNA replication inhibitor) and rotenone (a complex I inhibitor), induced aberrant migration of vascular smooth muscle cells (VSMC), possibly by impairment of Akt phosphorylation in the insulin signaling pathway (Ahn et al., 2010). Interestingly, a pan inhibitor of Akt, triciribin, reciprocally damaged mitochondrial function, implying that Akt may be the bidirectional communication point between the insulin signaling pathway and mitochondria. Therefore, overexpression of the constitutively active form of Akt (myr-Akt) normalized the mitochondrial stress-induced VSMC migra- tion and mitochondrial functions. These results lead us to propose that Akt may be a possible therapeutic target for treating insulin resistance-associated atherosclerosis.

\section{MITOCHONDRIAL MEDICINE}

All of the observations on the pathophysiology of the age-associated degenerative diseases share many common underlying energy metabolism and mitochondrial features (Wallace, 2005). Stimulation of mitochondrial biogenesis, increasing ATP production, and reducing ROS generation may have beneficial effects in age-related degenerative diseases (Kim et al., 2008). Unfortunately, as summarized in Table I, not many mitochondrial therapeutics have been reported, except for several vitamin cofactors and antioxidants, such as coenzyme Q10 (CoQ10), vitamin $\mathrm{C}$, uridine, and lipoic acid. However, treatment of patients with single metabolites or cofactors was often inefficient or yielded inconsistent results (Wallace et al., 2010). Furthermore, treatment for mitochondrial genetic diseases remains unsatisfactory even using high concentrations of the cofactors and antioxidants. Gene therapy, by manipulating mutant mtDNA and mtRNA, as well as the mitochondrial import of mtDNA gene products, raises many challenges for an in vivo application.

Given the mitochondrial pathophysiology of the diseases explained above, mitochondria represent an attractive therapeutic target to treat metabolic syndrome and neurodegenerative diseases. A brief review of present therapeutics would give some directions to develop "excellent mitochondrial medicine" to treat most mitochondria- defective diseases.

Coenzyme Q10, a component of OXPHOS, is decreased with aging. CoQ10 deficiency is also expected in the patients taking statins (HMG-CoA reductase inhibitors) to reduce blood cholesterol because statins decrease the supply of isoprene units which are necessary for CoQ10 synthesis. CoQ10 supplementation is very likely to benefit patients with mitochondrial defects as well as CoQ10 deficiency, since the increased CoQ10 may enhance the transfer of electrons by bypassing the complex I and complex II to complex III. By the same logic, succinate and vita$\min C$ are used to treat patients, but with varying degrees of success. However, the low bioavailability is a current obstacle in developing CoQ10 as a mitochondrial medicine due to its lipophilicity. Idebenone is a CoQ10 analog currently under development for cardiovascular and neurological disorders which has been met with limited success (Frantz and Wipf, 2010). MitoQ, another CoQ10 analog which is accumulating in mitochondria by covalent attach- 
Table I. List of mitochondrial therapeutics

\begin{tabular}{|c|c|c|c|}
\hline Agents & Indication/Applicable diseases & Mechanism & Feature \\
\hline \multicolumn{4}{|c|}{ Cofactors of OXPHOS for ATP production } \\
\hline CoQ10 & Mitochondrial disorders & Transfers electrons from complex I and II & \\
\hline MitoQ10 & Mitochondrial disorders & to complex III & \\
\hline Idebenone & Mitochondrial disorders & Mitochondrial targeting CoQ10 & \\
\hline Succinate & Complex I deficiency & $\begin{array}{l}\text { Transfers electrons from complex I and II } \\
\text { to complex III }\end{array}$ & \\
\hline Dimethylglycine & Mitochondrial disorders & Bypasses complex I & \\
\hline$\beta$-Hydroxybutyrate & PD & Transfers electrons to CoQ10 & \\
\hline Succinate plus CoQ10 & $\begin{array}{l}\text { Mitochondrial disorders, } \\
\text { CPEO }\end{array}$ & $\begin{array}{l}\text { Mitochondrial respiration and ATP synthesis } \\
\text { Bypasses complex I }\end{array}$ & \\
\hline \multicolumn{4}{|c|}{ Other neutriceuticals (vitamins or metabolic supplements) } \\
\hline Thiamine (vitamin B1) & $\begin{array}{l}\text { Pyruvate dehydrogenase } \\
\text { (PDH) deficiency }\end{array}$ & Cofactor of $\mathrm{PDH}$ variable & \\
\hline Riboflavin (vitamin B2) & Complex I and II deficiency & Increases complex I and II activities & \\
\hline Nicotinamide (vitamin B3) & MELAS & Increases nicotinamide adenine dinucleotide & \\
\hline Dichloroacetate & Severe lactic acidosis & Reduces lactic acidosis, stimulates PDH & \\
\hline Creatine monohydrate & Mitochondrial myopathy & Increases muscle phosphocreatine & \\
\hline Carnitine & Carnitine deficiency & $\begin{array}{l}\text { Increases carnitine level, ATP/ADP } \\
\text { regulation }\end{array}$ & \\
\hline L-arginine & MELAS & Increases nitric oxide production & \\
\hline Cystamine & $\mathrm{HD}$ & Inhibition of mitochondrial depolarization & \\
\hline Combinations of vitamines & Mitochondrial myopathy & & \\
\hline \multicolumn{4}{|c|}{ ROS scavengers and mitochondrial antioxidants } \\
\hline CoQ/Idebenone & Mitochondrial disorders & Antioxidant & \\
\hline Vitamin $\mathrm{E}$ & Mitochondrial disorders & Antioxidant & \\
\hline $\begin{array}{l}\text { Vitamin C (ascorbate)/ } \\
\text { vitamin K3 (menadione) }\end{array}$ & Mitochondrial disorders & Antioxidant, Bypass complex I, II, III & \\
\hline Lipoic acid & PDH deficiency, Obesity & Antioxidant, stimulates PDH & \\
\hline Triacetyluridine & Mitochondrial disorders & De novo pyrimidine biosynthesis & Animal models \\
\hline Melatonin & & Antioxidant & \\
\hline N-Acetylcysteine & & Antioxidant & In vitro studies \\
\hline \multicolumn{4}{|c|}{ Inhibition of the mIPTP and apoptosis } \\
\hline Cyclosporin A & $A D, P D, H D, A L S$ & Inhibits the mtPTP, calcineurin inhibitor & In vitro studies \\
\hline Nortriptyline & & Inhibits the mtPTP & Animal models \\
\hline Dimebon & $A D, P D$ & Inhibits the mtPTP & \\
\hline \multicolumn{4}{|c|}{ Enhancement of mitochondrial biogenesis } \\
\hline Sirtuin analogs & Mitochondrial disorders & Increase mitochondrial biogenesis & Animal models \\
\hline Bezafibrate & Mitochondrial disorders & Increase mitochondrial biogenesis & $\begin{array}{l}\text { In vitro studies, } \\
\text { Animal models }\end{array}$ \\
\hline $\begin{array}{l}\text { PPAR } \text { agonist } \\
\text { (rosiglitazone) }\end{array}$ & Metabolic syndrome & $\begin{array}{l}\text { Mitochondrial calcium and membrane } \\
\text { potential }\end{array}$ & \\
\hline Metformine & Metabolic syndrome & Increase mitochondrial biogenesis & In vitro studies \\
\hline Deferoxamine & & Mitochondrial gene regulation, metal chelator & Aging-related \\
\hline Taurine & Mitochondrial disorders & $\begin{array}{l}\text { Increase mitochondrial transcription, } \\
\text { mitochondrial tRNA synthesis }\end{array}$ & In vitro studies \\
\hline \multicolumn{4}{|l|}{ Diet and exercise } \\
\hline High-fat diet & Mitochondrial disorders & Increases electron transfer & \\
\hline Ketonic diet & $\begin{array}{l}\text { Mitochondrial disorders, } \\
\text { Intractable seizures }\end{array}$ & Bypasses glycolytic pathway & \\
\hline Endurance exercise & Mitochondrial myopathy & Aerobic training induces $\mathrm{OXPHOS}$ & \\
\hline Resistance exercise & Mitochondrial myopathy & $\begin{array}{l}\text { Activation and proliferation of satellite cells } \\
\text { in muscle, shifting of heteroplasmy }\end{array}$ & \\
\hline Calorie restriction & Aging & Increase mitochondrial biogenesis & \\
\hline
\end{tabular}

ment to a lipophilic triphenylphosphonium cation, is being evaluated to treat the mitochondrial dysfunction-related diseases. However, a large-scale clinical study of CoQ10 for PD demonstrated "non-effectiveness of CoQ10". The effects of other antioxidants or ROS scavengers used for treatment of mitochondrial diseases have been insufficient to improve the disease. Thus, increased efficacy is necessary, but yet to be demonstrated in clinical trials (Lee et al., 
2009; Wallace et al., 2010).

Insulin sensitizers, thiazolidinediones (synthetic PPAR- $\gamma$ agonists) and metformin, improve insulin resistance in various tissues. Their molecular mechanism for insulin-sensitizing activity may be, in part, accounted for through increased mitochondrial biogenesis through PGC-1 $\alpha$. They also reduce ROS production and stimulate AMPK. Besides pharmacological agents, aerobic exercise also stimulates mitochondrial biogenesis and glucose/lipid metabolism. Endurance exercise training increases mitochondrial size, number, and oxidative activity by activation of PGC-1 $\alpha$, NRF-1, TFAM, and AMPK, contributing to improved wholebody glucose metabolism. Adequate calorie restriction increases lifespan in organisms ranging from yeast to mammals and increases expression of UCP2 and -3 in human subjects. Calorie restriction increases mitochondrial biogenesis, oxygen consumption, ATP production, and expression of Sirt1 (NAD ${ }^{+}$-dependent deacetylase). Interestingly, a famous antioxidant, resveratrol, improves mitochondrial function and protects against metabolic diseases by activating Sirt1 and PGC-1 $\alpha$ (Lagouge et al., 2006). Sirt1 controls a variety of target proteins and analogs of Sirt1 activators have been developed to treat diabetes; some of them are under clinical trials (Milne et al., 2007). In turn, some mitochondria-targeted drug candidates are under development (Frantz and Wipf, 2010). In depth investigation is needed to design or develop safe and efficacious mitochondrial activators for treating or intervening in both agerelated degenerative mitochondrial diseases and the mitochondrial genetic diseases.

\section{ACKNOWLEDGMENTS}

This study was supported by FPR08A1-071 of 21C Frontier Functional Proteomics Project and NRF grants (20090084158, 20090084844, and 20090063278) from the Korean Ministry of Science \& Technology.

\section{REFERENCES}

Ahn, S. Y., Choi, Y. S., Koo, H. J., Jeong, J. H., Park, W. H., Kim, M., Piao, Y. and Pak, Y. K. (2010). Mitochondrial dysfunction enhances the migration of vascular smooth muscle cells via suppression of Akt phosphorylation. Biochim. Biophys. Acta. 1800, 275-281.

Barja, G. and Herrero, A. (2000). Oxidative damage to mitochondrial DNA is inversely related to maximum life span in the heart and brain of mammals. FASEB. J. 14, 312-318

Capaldi, R. A. (2000). The changing face of mitochondrial research. Trends Biochem. Sci. 25, 212-214.

Chan, D. C. (2006a). Mitochondria: dynamic organelles in disease, aging, and development. Cell 125, 1241-1252.
Chan, D. C. (2006b). Mitochondrial fusion and fission in mammals. Annu. Rev. Cell Dev. Biol. 22, 79-99.

Chen, H. and Chan, D. C. (2009). Mitochondrial dynamics-fusion, fission, movement, and mitophagy--in neurodegenerative diseases. Hum. Mol. Genet. 18, R169-176.

Cho, D. H., Nakamura, T., Fang, J., Cieplak, P., Godzik, A., Gu, Z. and Lipton, S. A. (2009). S-nitrosylation of Drp1 mediates beta-amyloid-related mitochondrial fission and neuronal injury. Science 324, 102-105.

Choi, Y. S., Hong, J. M., Lim, S., Ko, K. S. and Pak, Y. K. (2006). Impaired coactivator activity of the Gly482 variant of peroxisome proliferator-activated receptor gamma coactivator1alpha (PGC-1alpha) on mitochondrial transcription factor $A$ (TFAM) promoter. Biochem. Biophys. Res. Commun. 344, 708-712.

Choi, Y. S., Lee, K. U. and Pak, Y. K. (2004). Regulation of mitochondrial transcription factor $\mathrm{A}$ expression by high glucose. Ann. N Y Acad. Sci. 1011, 69-77.

Cortopassi, G. A., Shibata, D., Soong, N. W. and Arnheim, N. (1992). A pattern of accumulation of a somatic deletion of mitochondrial DNA in aging human tissues. Proc. Natl. Acad. Sci. U S A 89, 7370-7374.

Ekstrand, M. I., Falkenberg, M., Rantanen, A., Park, C. B. Gaspari, M., Hultenby, K., Rustin, P., Gustafsson, C. M. and Larsson, N. G. (2004). Mitochondrial transcription factor A regulates mtDNA copy number in mammals. Hum. Mol. Genet. 13, 935-944.

Falkenberg, M., Larsson, N. G. and Gustafsson, C. M. (2007). DNA replication and transcription in mammalian mitochondria. Annu. Rev. Biochem. 76, 679-699.

Frantz, M. C. and Wipf, P. (2010). Mitochondria as a target in treatment. Environ. Mol. Mutagen. 51, 462-475.

Fujitani, Y., Ueno, T. and Watada, H. (2010). Autophagy in health and disease. 4. The role of pancreatic beta-cell autophagy in health and diabetes. Am. J. Physiol. Cell Physiol. 299, C1-6.

Gaspari, M., Falkenberg, M., Larsson, N. G. and Gustafsson, C. M. (2004). The mitochondrial RNA polymerase contributes critically to promoter specificity in mammalian cells. EMBO. J. 23, 4606-4614.

Hoppins, S., Lackner, L. and Nunnari, J. (2007). The machines that divide and fuse mitochondria. Annu. Rev. Biochem. 76, 751-780.

Kim, J. A., Wei, Y. and Sowers, J. R. (2008). Role of mitochondrial dysfunction in insulin resistance. Circ. Res. 102, 401-414.

Kujoth, G. C., Hiona, A., Pugh, T. D., Someya, S., Panzer, K., Wohlgemuth, S. E., Hofer, T., Seo, A. Y., Sullivan, R., Jobling, W. A., Morrow, J. D., Van Remmen, H., Sedivy, J. M., Yamasoba, T., Tanokura, M., Weindruch, R., Leeuwenburgh, C. and Prolla, T. A. (2005). Mitochondrial DNA mutations, oxidative stress, and apoptosis in mammalian aging. Science 309, 481-484

Lagouge, M., Argmann, C., Gerhart-Hines, Z., Meziane, H., Lerin, C., Daussin, F., Messadeq, N., Milne, J., Lambert, P., Elliott, P., Geny, B., Laakso, M., Puigserver, P. and Auwerx, J. (2006). Resveratrol improves mitochondrial function and protects against metabolic disease by activating SIRT1 and PGC-1alpha. Cell 127, 1109-1122.

Lane, N. (2006a). Cell biology: power games. Nature 443, $901-$ 903. 
Lane, N. (2006b). Mitochondrial disease: powerhouse of disease. Nature 440, 600-602.

Larsson, N. G. (2010). Somatic mitochondrial DNA mutations in mammalian aging. Annu. Rev. Biochem. 79, 683-706.

Larsson, N. G. and Rustin, P. (2001). Animal models for respiratory chain disease. Trends Mol. Med. 7, 578-581.

Larsson, N. G., Wang, J., Wilhelmsson, H., Oldfors, A., Rustin, P., Lewandoski, M., Barsh, G. S. and Clayton, D. A. (1998) Mitochondrial transcription factor $A$ is necessary for mtDNA maintenance and embryogenesis in mice. Nat. Genet. 18, 231-236.

Lee, C. K., Allison, D. B., Brand, J., Weindruch, R. and Prolla, T. A. (2002). Transcriptional profiles associated with aging and middle age-onset caloric restriction in mouse hearts. Proc. Natl. Acad. Sci. U S A 99, 14988-14993.

Lee, H. K., Park, K. S., Cho, Y. M., Lee, Y. Y. and Pak, Y. K. (2005). Mitochondria-based model for fetal origin of adult disease and insulin resistance. Ann. N Y Acad. Sci. 1042 $1-18$.

Lee, H. K., Song, J. H., Shin, C. S., Park, D. J., Park, K. S., Lee, K. U. and Koh, C. S. (1998). Decreased mitochondrial DNA content in peripheral blood precedes the development of non-insulin-dependent diabetes mellitus. Diabetes Res. Clin. Pract. 42, 161-167.

Lee, J., Boo, J. H. and Ryu, H. (2009). The failure of mitochondria leads to neurodegeneration: Do mitochondria need a jump start? Adv. Drug Deliv. Rev. 61, 1316-1323.

Leone, T. C., Lehman, J. J., Finck, B. N., Schaeffer, P. J., Wende, A. R., Boudina, S., Courtois, M., Wozniak, D. F., Sambandam, N., Bernal-Mizrachi, C., Chen, Z., Holloszy, J. O., Medeiros, D. M., Schmidt, R. E., Saffitz, J. E., Abel, E. D., Semenkovich, C. F. and Kelly, D. P. (2005). PGC-1alpha deficiency causes multi-system energy metabolic derangements: muscle dysfunction, abnormal weight control and hepatic steatosis. PLoS Biol. 3, e101.

Liang, H. and Ward, W. F. (2006). PGC-1alpha: a key regulator of energy metabolism. Adv. Physiol. Educ. 30, 145-151.

Lim, S., Ahn, S. Y., Song, I. C., Chung, M. H., Jang, H. C., Park, K. S., Lee, K. U., Pak, Y. K. and Lee, H. K. (2009). Chronic exposure to the herbicide, atrazine, causes mitochondrial dysfunction and insulin resistance. PLoS One 4, e5186.

Lin, M. T. and Beal, M. F. (2006). Mitochondrial dysfunction and oxidative stress in neurodegenerative diseases. Nature 443, 787-795

Lustbader, J. W., Cirilli, M., Lin, C., Xu, H. W., Takuma, K., Wang, N., Caspersen, C., Chen, X., Pollak, S., Chaney, M., Trinchese, F., Liu, S., Gunn-Moore, F., Lue, L. F., Walker, D. G., Kuppusamy, P., Zewier, Z. L., Arancio, O., Stern, D., Yan, S. S. and Wu, H. (2004). ABAD directly links Abeta to mitochondrial toxicity in Alzheimer's disease. Science 304, 448-452.

MacAskill, A. F. and Kittler, J. T. (2010). Control of mitochondrial transport and localization in neurons. Trends Cell Biol. 20, 102-112.

Madamanchi, N. R. and Runge, M. S. (2007). Mitochondrial dysfunction in atherosclerosis. Circ. Res. 100, 460-473.

Matsushima, Y., Garesse, R. and Kaguni, L. S. (2004). Drosophila mitochondrial transcription factor B2 regulates mitochondrial DNA copy number and transcription in schneider cells. J. Biol. Chem. 279, 26900-26905.
McBride, H. M. (2008). Parkin mitochondria in the autophagosome. J. Cell. Biol. 183, 757-759.

Michikawa, Y., Mazzucchelli, F., Bresolin, N., Scarlato, G. and Attardi, G. (1999). Aging-dependent large accumulation of point mutations in the human mtDNA control region for replication. Science 286, 774-779.

Milne, J. C., Lambert, P. D., Schenk, S., Carney, D. P., Smith, J. J., Gagne, D. J., Jin, L., Boss, O., Perni, R. B., Vu, C. B., Bemis, J. E., Xie, R., Disch, J. S., Ng, P. Y., Nunes, J. J., Lynch, A. V., Yang, H., Galonek, H., Israelian, K., Choy, W., Iffland, A., Lavu, S., Medvedik, O., Sinclair, D. A., Olefsky, J. M., Jirousek, M. R., Elliott, P. J. and Westphal, C. H. (2007). Small molecule activators of SIRT1 as therapeutics for the treatment of type 2 diabetes. Nature 450, 712-716.

Narendra, D., Tanaka, A., Suen, D. F. and Youle, R. J. (2008). Parkin is recruited selectively to impaired mitochondria and promotes their autophagy. J. Cell Biol. 183, 795-803.

Okamoto, K. and Shaw, J. M. (2005). Mitochondrial morphology and dynamics in yeast and multicellular eukaryotes. Annu. Rev. Genet. 39, 503-536.

Park, J., Lee, S. B., Lee, S., Kim, Y., Song, S., Kim, S., Bae, E., Kim, J., Shong, M., Kim, J. M. and Chung, J. (2006). Mitochondrial dysfunction in Drosophila PINK1 mutants is complemented by parkin. Nature 441, 1157-1161.

Park, K. S., Nam, K. J., Kim, J. W., Lee, Y. B., Han, C. Y., Jeong J. K., Lee, H. K. and Pak, Y. K. (2001). Depletion of mitochondrial DNA alters glucose metabolism in SK-Hep1 cells. Am. J. Physiol. Endocrinol. Metab. 280, E1007-1014.

Park, K. S., Song, J. H., Lee, K. U., Choi, C. S., Koh, J. J., Shin, C. S. and Lee, H. K. (1999). Peripheral blood mitochondrial DNA content correlates with lipid oxidation rate during euglycemic clamps in healthy young men. Diabetes Res. Clin. Pract. 46, 149-154.

Park, K. S., Wiederkehr, A., Kirkpatrick, C., Mattenberger, Y., Martinou, J. C., Marchetti, P., Demaurex, N. and Wollheim, C. B. (2008). Selective actions of mitochondrial fission/fusion genes on metabolism-secretion coupling in insulin-releasing cells. J. Biol. Chem. 283, 33347-33356.

Patti, M. E., Butte, A. J., Crunkhorn, S., Cusi, K., Berria, R., Kashyap, S., Miyazaki, Y., Kohane, I., Costello, M., Saccone, R., Landaker, E. J., Goldfine, A. B., Mun, E., DeFronzo, R., Finlayson, J., Kahn, C. R. and Mandarino, L. J. (2003). Coordinated reduction of genes of oxidative metabolism in humans with insulin resistance and diabetes: Potential role of PGC1 and NRF1. Proc. Natl. Acad. Sci. U S A 100, 8466-8471.

Perry, G. M., Tallaksen-Greene, S., Kumar, A., Heng, M. Y., Kneynsberg, A., van Groen, T., Detloff, P. J., Albin, R. L. and Lesort, M. (2010). Mitochondrial calcium uptake capacity as a therapeutic target in the R6/2 mouse model of Huntington's disease. Hum. Mol. Genet. in press.

Petersen, K. F., Befroy, D., Dufour, S., Dziura, J., Ariyan, C., Rothman, D. L., DiPietro, L., Cline, G. W. and Shulman, G. I. (2003). Mitochondrial dysfunction in the elderly: possible role in insulin resistance. Science 300, 1140-1142.

Petersen, K. F., Dufour, S., Befroy, D., Garcia, R. and Shulman, G. I. (2004). Impaired mitochondrial activity in the insulinresistant offspring of patients with type 2 diabetes. N. Engl. J. Med. 350, 664-671.

Puigserver, P., Wu, Z., Park, C. W., Graves, R., Wright, M. and Spiegelman, B. M. (1998). A cold-inducible coactivator of 
nuclear receptors linked to adaptive thermogenesis. Cell 92, 829-839.

Saha, S., Guillily, M. D., Ferree, A., Lanceta, J., Chan, D., Ghosh, J., Hsu, C. H., Segal, L., Raghavan, K., Matsumoto, K., Hisamoto, N., Kuwahara, T., Iwatsubo, T., Moore, L., Goldstein, L., Cookson, M. and Wolozin, B. (2009). LRRK2 modulates vulnerability to mitochondrial dysfunction in Caenorhabditis elegans. J. Neurosci. 29, 9210-9218.

Schapira, A. H. (2006). Mitochondrial disease. Lancet. 368, 7082.

Silva, J. P., Kohler, M., Graff, C., Oldfors, A., Magnuson, M. A., Berggren, P. O. and Larsson, N. G. (2000). Impaired insulin secretion and beta-cell loss in tissue-specific knockout mice with mitochondrial diabetes. Nat. Genet. 26, 336-340.

Song, J., Oh, J. Y., Sung, Y. A., Pak, Y. K., Park, K. S. and Lee, H. K. (2001). Peripheral blood mitochondrial DNA content is related to insulin sensitivity in offspring of type 2 diabetic patients. Diabetes Care 24, 865-869.

Trifunovic, A., Hansson, A., Wredenberg, A., Rovio, A. T., Dufour, E., Khvorostov, I., Spelbrink, J. N., Wibom, R., Jacobs, H. T. and Larsson, N. G. (2005). Somatic mtDNA mutations cause aging phenotypes without affecting reactive oxygen species production. Proc. Natl. Acad. Sci. U S A 102, 17993-17998.

Trifunovic, A., Wredenberg, A., Falkenberg, M., Spelbrink, J. N. Rovio, A. T., Bruder, C. E., Bohlooly, Y. M., Gidlof, S., Oldfors, A., Wibom, R., Tornell, J., Jacobs, H. T. and Larsson, N. G. (2004). Premature ageing in mice expressing defective mitochondrial DNA polymerase. Nature 429, 417-423.

Twig, G., Elorza, A., Molina, A. J., Mohamed, H., Wikstrom, J. D., Walzer, G., Stiles, L., Haigh, S. E., Katz, S., Las, G., Alroy, J., Wu, M., Py, B. F., Yuan, J., Deeney, J. T., Corkey, B. E. and Shirihai, O. S. (2008). Fission and selective fusion govern mitochondrial segregation and elimination by autophagy. EMBO J. 27, 433-446.

Wagner, B. K., Kitami, T., Gilbert, T. J., Peck, D., Ramanathan, A., Schreiber, S. L., Golub, T. R. and Mootha, V. K. (2008). Large-scale chemical dissection of mitochondrial function. Nat. Biotechnol. 26, 343-351.

Wallace, D. C. (2005). A mitochondrial paradigm of metabolic and degenerative diseases, aging, and cancer: a dawn for evolutionary medicine. Annu. Rev. Genet. 39, 359-407.

Wallace, D. C., Fan, W. and Procaccio, V. (2010). Mitochondrial energetics and therapeutics. Annu. Rev. Pathol. 5, 297-348.

Wang, X. and Schwarz, T. L. (2009). The mechanism of $\mathrm{Ca}^{2+}$ dependent regulation of kinesin-mediated mitochondrial motility. Cell 136, 163-174.

Wang, Y., Michikawa, Y., Mallidis, C., Bai, Y., Woodhouse, L., Yarasheski, K. E., Miller, C. A., Askanas, V., Engel, W. K., Bhasin, S. and Attardi, G. (2001). Muscle-specific mutations accumulate with aging in critical human mtDNA control sites for replication. Proc. Natl. Acad. Sci. U S A 98, 4022-4027.

Yankner, B. A., Lu, T. and Loerch, P. (2008). The aging brain. Annu. Rev. Pathol. 3, 41-66. 